\title{
Spontaneous in planta changes in fungal endophytes impact symbiosis
}

\author{
W.R. SIMPSON ${ }^{1}$, M.J. CHRISTENSEN ${ }^{1}$, R.D. JOHNSON ${ }^{1}$ and J.SCHMID ${ }^{2}$ \\ ${ }^{I}$ Forage Improvement Section, AgResearch Limited, Grasslands Research Centre, Tennent Drive, Private Bag 11008, Palmerston \\ North, New Zealand \\ ${ }^{2} I M B S$, Turitea Campus, Massey University, Palmerston North \\ wayne.simpson@agresearch.co.nz
}

\begin{abstract}
The symbiosis between the asexual Ascomycetous fungi of the genus Neotyphodium and host grasses is considered to be symptomless. Symbioses involving Epichloë can show symptoms but this is restricted to the formation of stroma on floral structures. Fungal mycelium of both Epichloë and Neotyphodium endophytes occupy the intercellular space of plant tissues with no breach of host cell walls. Research involving these endophytes and their host grass symbioses relies on staining of host tissues and microscopic examination, immuno-detection or in-vitro isolation to determine infection status. This work reports on the observation of two independent instances of spontaneous change in the endophyte fungus that manifests as changes in the host grass vegetative morphology; one involving a genetically modified Epichloë festucae inoculated into a perennial ryegrass (Lolium perenne) population and the other a resident wild type Neotyphodium lolii. The relationship between wild type and variant fungus is confirmed as is the connection between variant fungus and host morphology. This work demonstrates that spontaneous in-planta changes in fungal endophytes occur and that these can impact on fungus/host grass symbioses.
\end{abstract}

Keywords: Neotyphodium, Epichloë, symbiosis, endophyte, perennial ryegrass

\section{Introduction}

Fungal endophytes of grasses, like all organisms, are subject to change both at a genetic and epigenic level and yet they maintain mutually advantageous symbioses with their hosts. First principles tell us that any changes in the endophyte that adversely affect the symbiosis will be selected against and therefore the evidence of such changes in the population is likely to be ephemeral. Here we describe the host plant effects of two examples of spontaneous in-planta change of fungal endophytes, one in Neotyphodium lolii and one in Epichloë festucae.

The sexual Epichloë species exhibit overt signs of their presence within the host when they produce stromata on reproductive tillers (Schardl et al. 2004). Asexual Neotyphodium do not produce stromata on reproductive tillers and infected plants are in no way observably different from uninfected notwithstanding any effects of biotic and abiotic stresses in the field. To positively ascertain infection status of grasses forming symbioses with Neotyphodium endophytes such as the perennial ryegrass endophyte N.lolii and E. festucae, it is necessary either to stain tissue and observe mycelium directly or to use an immunological technique to observe the presence of fungus indirectly.

The 'invisibility' of these endophytic fungi reflects the level of compatibility of the union of these symbionts.

A previous report described a deviation from this asymptomatic presence of Neotyphodium endophyte where a cultured novel N.lolii strain, AR5, induced a change in host grass phenotype (Simpson et al. 2004). Work performed in this study demonstrates that the host effects in this case are not due to a variant of the novel AR5 endophyte but to a variant, P1f, of the resident endemic N.lolii, P41f, that is extant in the population. An implication of this finding is that the change in the fungus has occurred in-planta with no opportunity for change within the unnatural environment of a Petri plate.

We also report on one other independent observation of spontaneous in-planta change in the endophytic fungus that results in a perturbation of the symbiosis involving a genetically modified E.festucae, F11 Nc25 (Johnson et al. 2007), and show that this change is stable in the fungus allowing transfer of the host altering effect to other populations via artificial infection.

\section{Methods}

Fungal isolation, seedling preparation and inoculation

Fungus was isolated from endophyte-infected plants by surfacesterilising plant tissue and culturing on antibiotic potato dextrose agar (ABPDA). Seed was surface-sterilised and germinated in the dark on $4 \%$ water agar and resulting seedlings inoculated as described by Latch and Christensen (1985).

\section{Identification of infected plants}

Inoculated seedlings were incubated in the dark for 7 days then placed under white fluorescent tubes for 7 days before being removed from Petri plates and planted into potting mix. Plants were grown for approximately 6 weeks before being assayed for endophyte infection using an immuno-assay as described by Hahn et al. (2003).

Some plants were examined by removing epidermal strips and staining with aniline blue and examining using a compound microscope at $100 \mathrm{X}$ magnification.

\section{Endophyte elimination}

Intact tillers, including roots, were removed from infected plants and washed thoroughly to remove potting soil then immersed in a Benlate solution $(2 \mathrm{~g} / \mathrm{L}$ ) for $3+$ hours. Tillers were planted in river sand in pots without drainage and watered to saturation with the same Benlate solution; watered pots were weighed and regularly watered to weight with fresh water only (to maintain original Benlate concentration) over a period of approximately 6 weeks.

\section{SSR}

For each analysis two tillers cut at soil level were trimmed to ca. $3 \mathrm{~cm}$ length and transferred to Q-BIOgene DNA extraction vials. Extracted DNA was amplified by PCR using primers flanking simple sequence repeats (SSRs) that are known to be polymorphic across strains of Neotyphodium endophyte (Moon et al. 1999).

\section{Alkaloid determination}

Herbage samples were cut to $5 \mathrm{~mm}$ of soil level and after removal of dead material divided into leaf blade and pseudostem portions prior to freeze drying. Samples were milled in a modified volume reduced domestic coffee grinder.

Ergovaline, combined with its isomer ergovalinine, was estimated by minor modifications of the method of Spiering et al. (Spiering et al. 2002) using 50mg samples extracted with $1 \mathrm{ml}$ of solvent. Solid residue was removed from the extract by 
Figure 1 Separated tillers of perennial ryegrass. Left: infected with endemic $N$. lolii showing normal phenotype. Right: infected with $N$. lolii variant fungus showing small tillered, fine leaved phenotype.

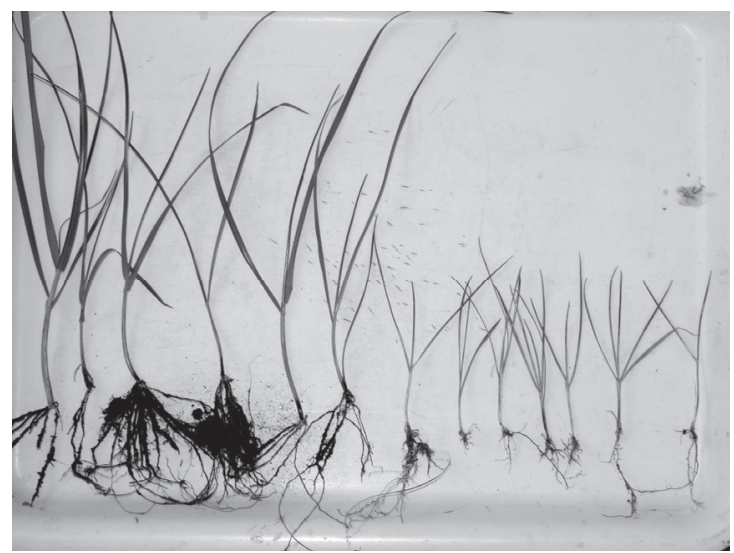

Figure 3 Mycelium of N.lolii P41f emerging from normal phenotype perennial ryegrass tissue on ABPDA.

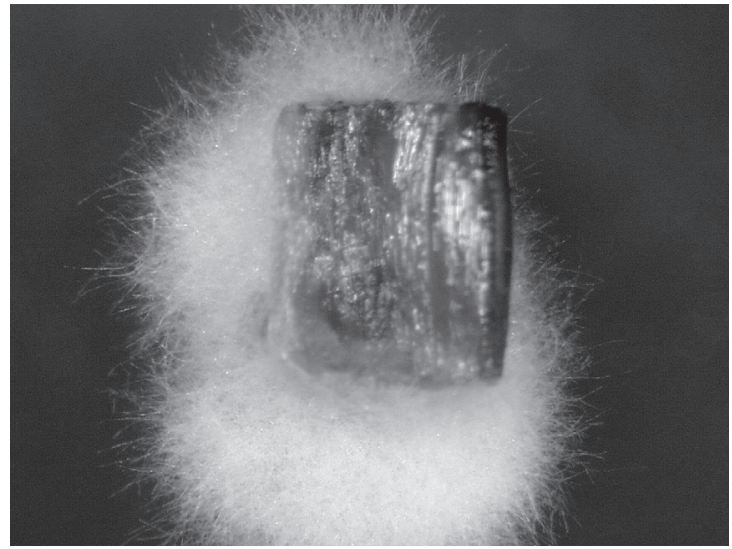

centrifugation. Peramine was estimated on the same extract using a different HPLC system also based upon Spiering et al. (Spiering et al. 2002).

Lolitrem B was estimated by a simplification of the method of Gallagher et al. (Gallagher et al. 1985). Samples (50mg) were extracted with $1 \mathrm{ml}$ of dichloroethane - methanol (9:1 by volume) for $1 \mathrm{hr}$ with mixing at ambient temperature and then centrifuged to remove solid residues prior to direct HPLC. Peak areas were compared to an external standard of lolitrem B.

\section{Results and Discussion}

\section{$N$. lolii fungus P41f is wild-type of P1f variant}

Alkaloid analysis of the parent plant of N.lolii AR5 (AR5pp) shows expression of peramine and ergovaline but not lolitrem B. Plant P41 hosting the endophyte P41f was positive for peramine, ergovaline and lolitrem B. Plant P1 hosting the variant fungus P1f was positive for peramine and ergovaline only, suggesting that AR5 is the wild-type of the variant fungus, however, SSR analysis shows that this is not the case.
Figure 2 Small stunted tillers develop on one side of a perennial ryegrass plant infected with Epichloë festucae strain FL1 NC25.

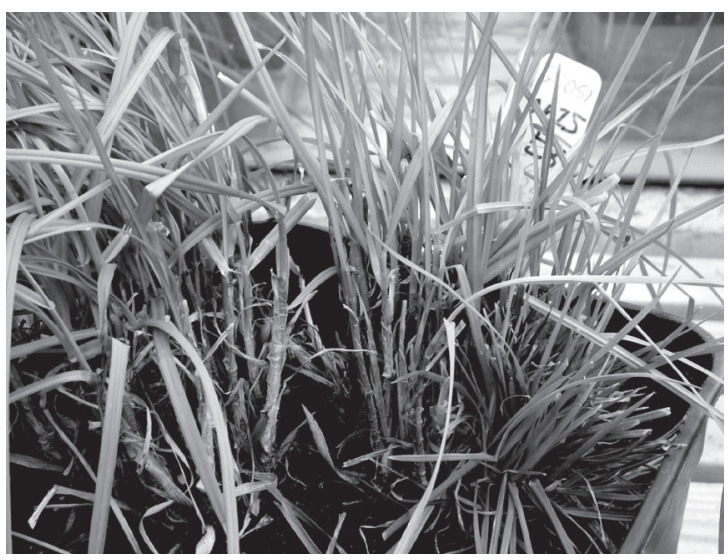

Figure 4 Mycelium of N.lolii variant fungus P1f from atypical morphology perennial ryegrass on ABPDA.

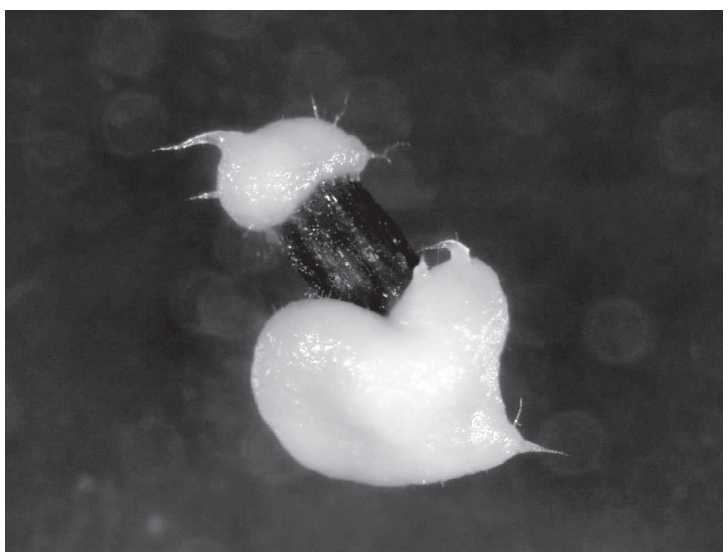

Analysis of plant material representing AR5pp, P1 and P41 all showed a 177 peak at the B10 locus. At the B11 locus AR5pp showed a 238 peak while both P1 and P41 showed a 177 peak. SSR data indicate that P41f is the wild-type of P1f but no lolitrem $\mathrm{B}$ has been detected in a P1f symbiosis.

Endophyte-free populations infected with P1f and variant FI1 Nc25 show altered phenotype

P1f was isolated and used in reinfection studies using both a heterogeneous out-crossing population and clonal plantlets from a single perennial ryegrass genotype to demonstrate a link between the fungus and the effects on the host grass. All seedlings from the endophyte-free population infected with P1f displayed the fine leaved phenotype (Fig. 1) previously described (Simpson et al. 2004) and the only clone infected with P1f was also fine leaved.

Plants infected with cultured AR5 or with P41f had a normal phenotype that did not differ from uninfected individuals from the same host population. 
The seedlings of perennial ryegrass infected with the genetically modified E. festucae strain F11 Nc25 were initially all normal phenotype. After a period of time one of the infected plants developed stunted tillers to one side (Fig. 2). Both normal and stunted tillers were examined and confirmed to be endophyteinfected. Fungal isolates from normal and stunted tillers were reinfected into an endophyte-free population of perennial ryegrass. Plants infected from these inoculations reproduced the effects: fungus from normal tillers gave rise to infected seedlings with a typical phenotype and fungus from stunted tillers gave rise to infected seedlings with a stunted phenotype.

In addition, the fine-leaved P1 plants were treated with fungicide to eliminate the endophyte fungus P1f; the endophytefree clones obtained all had a normal phenotype. It is clear from these results that the $N$.lolii fungus is responsible for the change in host phenotype.

P1f shows altered morphology in culture, Fl1 Nc25 variant similar to source fungus

Fungus isolated from AR5pp and P41 was typical in culture with a proliferation of aerial hyphae (Fig. 3) but P1f was not, with hyphae forming an aggregated mass with a smooth moist appearance (Fig. 4). Fungus isolated from F11 Nc25-infected normal tillers and that isolated from stunted tillers, produced colonies within the range of what might be considered typical for E.festucae Fl1.

Variant F11 Nc25 and P1f have in planta phenotypes different from their respective wild-types and from each other

Epidermal strips from variant F11 Nc25 plants stained with aniline blue show a proliferation of branched hyphae that contrasts with the moderate levels of largely unbranched hyphae in wildtype F11 Nc25.

Stained epidermal strips of P1 revealed extremely sparse distribution of otherwise typical hyphae. This result is interesting in that it demonstrates that it is not necessary to have a proliferation of mycelium to effect a change in host plant architecture.

Plants infected with P1f or Fl1 Nc25 have not produced seed When plants infected with P41 f and P1f were vernalised with a view to harvesting seed only the P41f-infected plants produced seed, P1f-infected plants did not produce any floral structures at all. There has not been any evidence of flowering or seed production in the Nc25 stunted plants, indeed the plants are very weak vegetatively and tend to undergo decline in the glasshouse.

\section{P1f and Fl1 Nc25 variant a result of in planta change}

Both the N.lolii $\mathrm{P} 41 \mathrm{f}$ and the E. festucae F11 Nc25 have undergone a change in planta that has resulted in a change in the phenotype of hosts infected with these variant fungi. This effect persists over time within the host and is a characteristic of the fungi that is maintained through culturing and re-infection of new hosts.

\section{ACKNOWLEDGEMENTS}

We would like to thank Marty Faville for SSR analysis and Brian Tapper and Liz Davies for alkaloid analyses.

\section{REFERENCES}

Gallagher, R.T.; Hawkes, A.D.; Stewart, J.M. 1985. Rapid determination of the neurotoxin lolitrem $B$ in perennial ryegrass by high-performance liquid chromatography with fluorescence detection. Journal of Chromatography 321: 217-226.

Hahn, H.; Huth, W.; Schoberlein, W.; Diepenbrock, W. 2003. Detection of endophytic fungi in Festuca spp. by means of tissue print immunoassay. Plant Breeding 122: 217-222.

Johnson, R.D.; Borchert, S.; Christensen, M. J.; Johnson, L.J.; Koulman, A.; van Gils, M.J.; Bryan, G.T. 2007. A gene identified from Neotyphodium lolii is expressed only in planta and regulates the biosynthesis of a putative oligopeptide secondary metabolite. pp. 485 In: Proceedings of the 6th International Symposium on Fungal Endophytes of Grasses. Eds. Popay, A.J.: Thom, E.R. Grassland Research \& Practice Series 13. New Zealand Grassland Association.

Latch, G.C.M.; Christensen, M.J. 1985. Artificial infection of grasses with endophytes. Annals of Applied Biology 107: 17-24.

Moon, C.D.; Tapper, B.A.; Scott, B. 1999. Identification of epichloë endophytes in planta by a microsatellite-based PCR fingerprinting assay with automated analysis. Applied and Environmental Microbiology 65: 1268-1279.

Schardl, C.L.; Spiering, M.J.; Leuchtmann, A. 2004. Symbioses of grasses with seedborne fungal endophytes. Annual Review of Plant Biology 55: 315-340.

Simpson, W.R; Christensen, M.J; Tapper, B.A 2004. Mutation in Fungal Endophyte Alters the Phenotype of Symbiont Host Plant. Poster 204 In: Proceedings of the 5th International Symposium on Neotyphodium/Grass Interactions. Eds. Kallenbach, R.; Rosenkrans, C.J.; Lock, T.R. Fayetteville, Arkansas.

Spiering, M.J.; Davies, E.; Tapper, B.A.; Schmid, J.; Lane, G.A. 2002. Simplified extraction of ergovaline and peramine for analysis of tissue distribution in endophyte-infected grass tillers. Journal of Agricultural and Food Chemistry 50: 5856. 